\title{
Thermal patterns of squamous cell carcinoma and actinic tumours
}

\author{
by C. Magalhaes*** , J. Mendes ${ }^{* *}$ and R. Vardasca****** \\ * INEGI - Institute of Science and Innovation in Mechanical and Industrial Engineering, Campus da FEUP, R. \\ Dr. Roberto Frias 400, 4200-465 Porto, Portugal. \\ ** Faculdade de Engenharia, Universidade do Porto, Rua Dr. Roberto Frias S/N, 4200-465 Porto, Portugal, \\ cmagalhaes@fe.up.pt / up201607752@fe.up.pt \\ *** ISLA Santarém, Largo Cândido dos Reis, 2000-241 Santarém, Portugal
}

\begin{abstract}
Squamous cell carcinoma (SCC) is often mistaken with its precursor lesion actinic keratosis (AK) due to their visual apparent resemblance. The current detection process presents faults and requires more information to improve the differential diagnosis of these skin tumour types. Infrared thermal (IRT) is able to detect skin surface temperature variations caused by physiological mechanisms, being a good candidate for its assessment. The aim of this research was to use IRT imaging to evaluate 80 patients with SCC and AK characteristic thermal patterns from image processing strategies. Clear differences were encountered between the thermal curves of the two tumour types, indicating that the applied methodology should be further explored for computer aided diagnosis accommodating machine learning methods.
\end{abstract}

\section{Introduction}

Non-melanoma skin cancer (NMSC) is the number one occurring malignant neoplasia type in Caucasian individuals, with over 1 million new cases worldwide only in 2018 [1], [2]. Its main subtypes are Basal Cell Carcinoma (BCC) and Squamous Cell Carcinoma (SCC), being the last harder to diagnosis due to its anatomical resemblance with its precursor lesion: Actinic Keratosis (AK) [3]. The detection strategy focuses on lesion monitoring over a period of time to assess its evolution. If the tumour is suspected to be malignant, histopathological tests are performed to confirm or refute the diagnosis. However, the early identification of SCC through a clinical evaluation can be ineffective and delayed when performed by physicians that fail to identify malignant traits due to limited experience. This can lead to lesion worsening, demanding harshest treatments and higher healthcare costs. Hence, an additional aid beyond visual features is of interest.

Infrared thermal (IRT) imaging is a non-ionizing, non-contact, non-invasive and fast technique that is able to detect skin surface temperature variations [4], [5]. When the formation of a skin tumour occurs, physiological mechanisms are disrupted in lesion site, causing shifts in temperature values that are passible of being assessed with this imaging method [6]. Its implementation can be static, or dynamic (through the application of a thermal, chemical or mechanical stimulus to the affected area), involving the latter the stimulation of lesion site to enhance underlying features, being the whole procedure object of IRT screening.

The aim of this work was to assess static and dynamic IRT imaging to evaluate the characteristic thermal patterns of SCC and AK lesions.

\section{Methodology}

This research was carried at Instituto Português de Oncologia do Porto Francisco Gentil, E.P.E, being the population group composed by patients of the Plastic and Reconstructive Surgery department. Every participant was of legal age. Patients that were considered mentally unfit or legally unable to consent its participation in the research project could choose to be represented by a legal representative. Each subject needed to present, at least, one possible neoplastic SCC or AK lesion for inclusion in this research and non-cooperating individuals, were discarded

With the approval of the ethics committee, SCC and AK thermograms were collected using the thermal camera FLIR (Wilsonville, OR, USA) E60sc. The calibration of the thermal camera was performed in advance with a calibration source, blackbody ISOTECH HYPERION R 982, using an controlled ambient temperature $\left(21 \pm 1^{\circ} \mathrm{C}\right)$ and relative humidity $(\leq 50 \%)$, which were monitored with the hygrometer TESTO 175 H1 [7], [8]. The thermal stimulation of the leased site was performed with an aluminium disc (diameter $=50 \mathrm{~mm}$, thickness $=20 \mathrm{~mm}$ ). The selection of this particular metal is justified by the elevated thermal conductivity that it presents, e. g., $237 \mathrm{~W} \mathrm{cm-1} \mathrm{K-1} \mathrm{at} \mathrm{298,2} \mathrm{K} \mathrm{[9],} \mathrm{providing} \mathrm{a} \mathrm{high} \mathrm{heat} \mathrm{transfer}$ rate across the disc.

The image acquisition protocol started with a 10-minute period of acclimatization to examination room conditions of the participants. Before the application of the thermal stimulus, a baseline thermogram was recorded (Image X). Then, a cold stimulus (through conduction with the aluminium disc) was applied to the tumour site during 1 minute and a new image was captured immediately after (Image 0). A 5 min thermal recovery period followed, were an image was collected per minute (Image 1, 2, 3, 4 and 5), being the thermal recovery process characterized by five thermograms. In total of 80 skin tumours were assessed, being 51 SCC and 29 AK. 


\subsection{Image Analysis}

The collected thermal images were firstly converted to .mat files with the software tool FLIR ThermaCAM Researcher Professional 2.10 and analysed in MatLab (Natick, Massachusetts, USA) 2018a.

Before the collection of thermal data, all lesions were selected by a user and cropped using a 40 by 40 -pixel window.

The static image analysis consisted of the gathering of the temperature values stored in the two main diagonals of the $40 \times 40$ area. The values of each diagonal were multiplied to attain a temperature profile representative of the skin tumour under assessment. The average lesion temperature and standard deviation of temperature of the 40x40 area were also collected for each skin tumour. Only image $X$ was used for this stage.

For the dynamic analysis all images were used. The difference between the maximum temperature of a 5-pixel area located in the inner tumour region $(20 \times 20)$ and the minimum temperature of 5-pixel area on its periphery was calculated for each image. The average lesion temperature and standard deviation of temperature of the $40 \times 40$ area were also collected for each skin tumour. The temperature differences, average lesion temperature and standard deviation of lesion temperature of each image were compared to assess changes during lesion thermal recovery.

Table 1. Static and Dynamic parameters collected for each skin lesion.

\begin{tabular}{|c|c|}
\hline \multicolumn{2}{|c|}{ Thermal parameter } \\
\hline \multirow{3}{*}{$\begin{array}{c}\text { Static } \\
(\text { Image X) }\end{array}$} & Thermal profile \\
\hline & Average lesion of temperature \\
\hline & Standard deviation of temperature \\
\hline \multirow{3}{*}{$\begin{array}{c}\text { Dynamic } \\
\text { (Image } \mathrm{X}, 0,1,2,3,4,5)\end{array}$} & Temperature Differences \\
\hline & Average lesion of temperature \\
\hline & Standard deviation of temperature \\
\hline
\end{tabular}

\section{Results and Discussion}

The results of the thermal analysis of the 80 skin tumours (51 SCC and 29 AK) are included in Fig. 1.

Both SCC and AK displayed static hypothermic profiles that overlap in the central region (Fig. 1A). The hypothermic valley of squamous cell carcinoma is more pronounced with higher slopes, resulting in lower temperatures when compared to actinic tumours. The accentuated concavity encountered in squamous cell carcinoma (SCC) tumours can be justified by the characteristic anatomy of this type of skin cancer. In fact, SCC tend to grow on height, due to the accumulation of a crust in the lesion site [3]. This structure causes an insulated environment, leading to the depression verified in the resultant temperature profile. The overlap of SCC and AK in the central region indicates that the differentiation of these tumours might only be achievable when focusing on peripheral temperature values. Since actinic tumours are precursor lesions of SCC, the valley found for this type of skin neoplasms is justified by the same anatomical alterations. However, the thermal insulation process is weaker due to the small thickness of the formed crust, making the thermal depression of AK less accentuated. This is also verified in the overall average lesion temperature (Fig. 1B), since squamous cell carcinomas presented lower temperatures values, probably due to the insulation crust encountered on the tumour area. The rough texture of the SCC crust results in increased image noise upon thermogram acquisition, so the higher value of standard deviation encountered, was also expected (Fig. 1C).

The thermal recovery curves of SCC and AK showed clear differences, particularly in the moment immediately after cooling (Fig 1D, E, F). Despite presenting a much more accentuated temperature difference after lesion stimulus, the SCC lesions show a smaller decrease in average lesion temperature when compared to AK tumours (Fig 1E). Additionally, the AK seemed to reach thermal equilibrium faster. This is supported by the anatomical features previously described, indicating that the leased area of the SCC delays thermal recovery. 
A

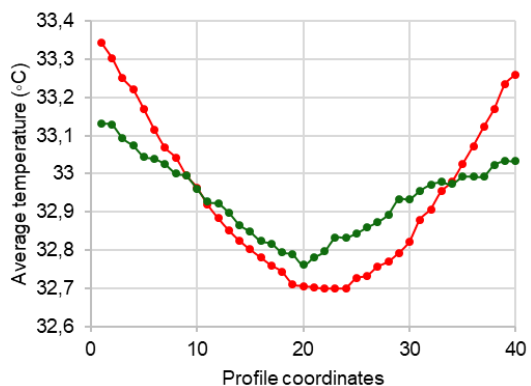

B

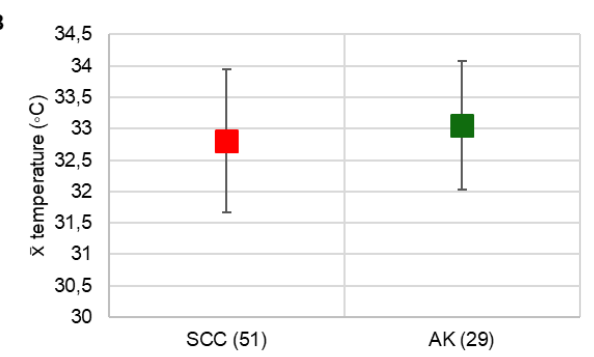

C

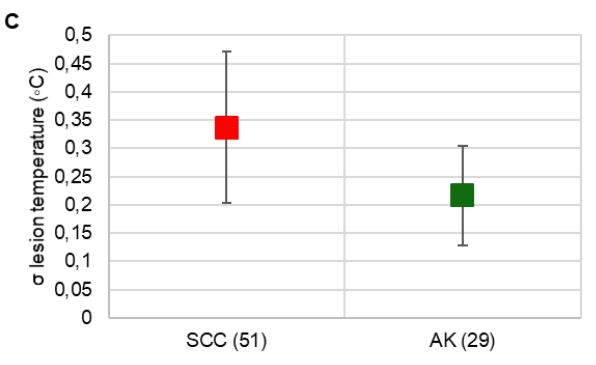

$\rightarrow$ SCC $(51)$ $\rightarrow$ AK (29)

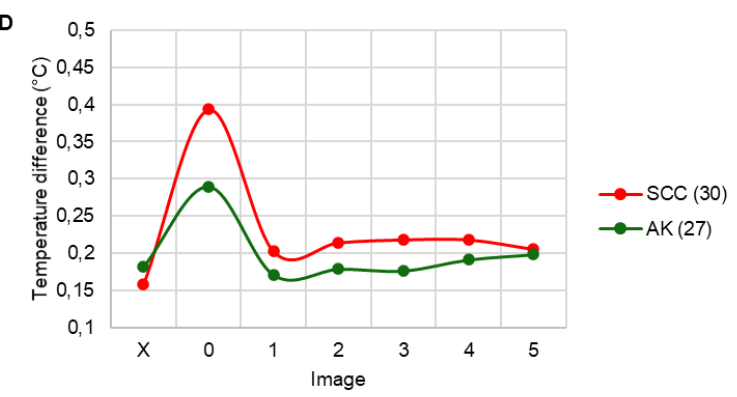

E
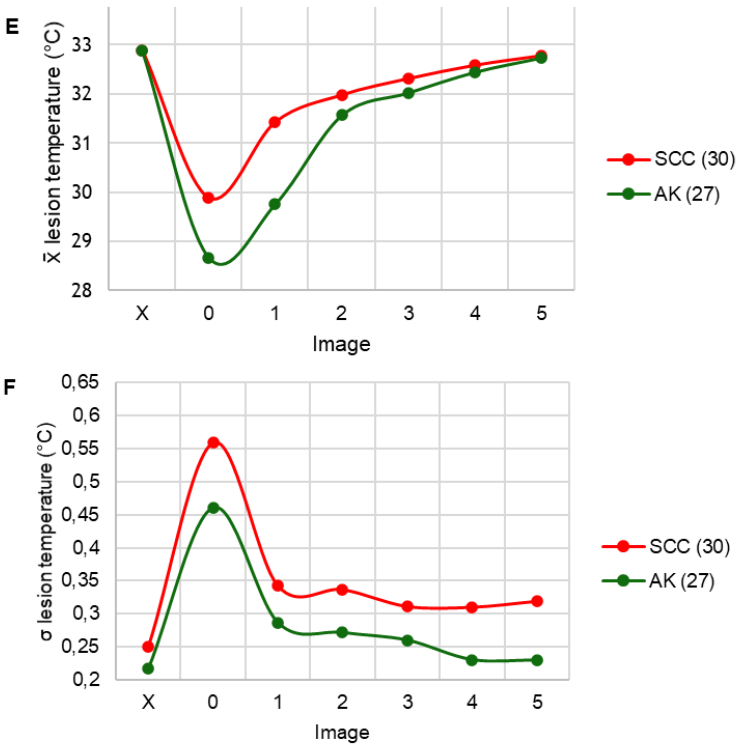

Fig. 1. Static image analysis: $A$ - Thermal curve of SCC and AK; B - Average lesion temperature; $C-A v e r a g e$ standard deviation of temperature; $D$ - Maximum temperature differences of SCC and AK during thermal recovery; $E$ Average lesion temperature during thermal recovery; $F$ - Average standard deviation of temperature during thermal recovery.

\section{Conclusions}

This research was performed with the aim of evaluating SCC and AK thermal patterns through the use of infrared thermal (IRT) imaging. The thermal images were collected in a medical setting and the static and dynamic IRT imaging approach allowed the collection of different features characteristic of the evaluated tumours. The analysis of the constricted profiles indicates that the distinction of benign actinic lesions from malignant squamous cell tumours is possible, showing the potential of this imaging technique for an early detection of SCC lesions. To test this hypothesis and facilitate the second opinion for physicians, this methodology should be further explored with the use of IRT parameters as inputs for computer aided diagnosis systems through the usage of machine learning methods.

\section{ACKNOWLEDGMENTS}

The authors gratefully acknowledge the funding of Project LAETA - UIDB/50022/2020 and the PhD Scholoarship from Fundação para a Ciência e a Tecnologia - SFRH/BD/144906/2019.

\section{REFERENCES}

[1] A. Lomas, J. Leonardi-Bee, and F. Bath-Hextall, "A systematic review of worldwide incidence of nonmelanoma skin cancer," Br. J. Dermatol., vol. 166, no. 5, pp. 1069-1080, 2012, doi: 10.1111/j.1365-2133.2012.10830.x.

[2] F. Bray, J. Ferlay, I. Soerjomataram, R. L. Siegel, L. A. Torre, and A. Jemal, "Global cancer statistics 2018: GLOBOCAN estimates of incidence and mortality worldwide for 36 cancers in 185 countries," CA. Cancer J. Clin., 
vol. 68, no. 6, pp. 394-424, 2018, doi: 10.3322/caac.21492.
R. A. Schwartz, "Squamous Cell Carcinoma," in Skin Cancer: Recognition and Management, 2nd ed., Newark, New Jersey: Blackwell Publishing, 2008, pp. 47-65.

[4] E. F. J. Ring and K. Ammer, "Infrared thermal imaging in medicine," Physiol. Meas., vol. 33, no. 3, pp. R33-R46, Mar. 2012, doi: 10.1088/0967-3334/33/3/R33

[5] K. Ammer and F. Ring, The Thermal Human Body. 2019.

[6] G. Garusi, "Thermography in the Study of Superficial and Semisuperficial Neoplastic Processes," Tumori J., vol. 56, no. 3, pp. 149-163, May 1970, doi: 10.1177/030089167005600302.

[7] K. Ammer, "The Glamorgan Protocol for recording and evaluation of thermal images of the human body," Thermol. Int., vol. 18, no. 4, pp. 125-129, 2008.

[8] E. Ring and K. Ammer, "The technique of infrared imaging in medicine," in Infrared Imaging, IOP Publishing, 2015, pp. $1-10$

[9] J. E. Hatch, "Properties of pure aluminum," in Aluminum: Properties and Physical Metallurgy, J. E. Hatch, Ed. ASM International, 1984, pp. 1-24. 